\title{
Diagnóstico participativo y prácticas pre-profesionales en comunidades de El Crucero, Managua, Nicaragua
}

\section{Participatory diagnosis and pre-professional practices in communities of El Crucero, Managua, Nicaragua}

\author{
Álvaro Benavides González ${ }^{1}$, Henry Alberto Duarte-Canales², Juan Carlos Morán Centeno ${ }^{2}$ \\ ${ }^{1}$ MSc. Ex-Coordinador e Investigador DEPARTIR / ORCID: https://orcid.org/0000-0002-0596-690X / alvaro.benavides@ci.una.edu.ni \\ ${ }_{2}^{2}$ MSc. Ex-Investigador de Campo DEPARTIR / ORCID: https://orcid.org/0000-0003-3515-845X / hduarte@ci.una.edu.ni / ORCID: https:// \\ orcid.org/0000-0001-6135-7271 / juan.moran@ci.una.edu.ni \\ Universidad Nacional Agraria, Facultad de Agronomía.
}

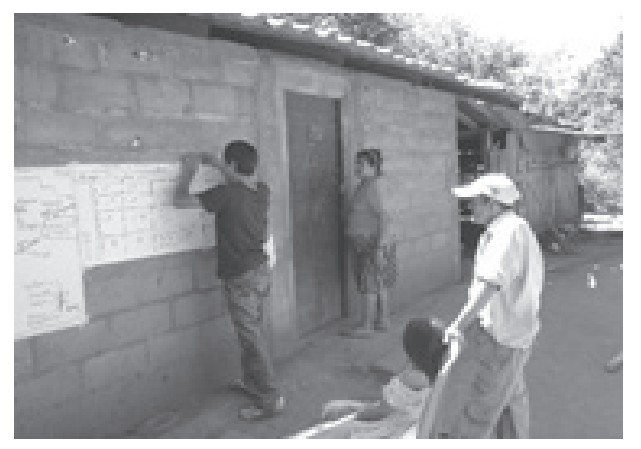

\begin{abstract}
RESUMEN
El programa para el Desarrollo Participativo Integral Rural (DEPARTIR) trabajó con familias, estudiantes y docentes investigadores de la Universidad Nacional Agraria (UNA), Universidad Agropecuaria de Viena (BOKU) y la Casa de los Tres Mundos de Granada (CTM) en comunidades rurales de Nicaragua con el apoyo del Programa Austriaco de Educación Superior e Investigación (APPEAR), así como la Organización de las Naciones Unidas para la Alimentación y la Agricultura (FAO). Durante el período 2006-2015 el programa tuvo intervención en 17 comunidades, desarrollado siete ediciones de diagnósticos y priorizadas propuestas en conjunto con las familias. El objetivo de este estudio fue comunicar la apreciación y valoración del curso teórico-práctico desarrollado por DEPARTIR a estudiantes y analizar información básica recopilada por estudiantes en cinco comunidades rurales de El Crucero, Managua. Fueron aplicadas encuestas en la unidad familiar de producción (UFP) para conocer las condiciones socieconómicas con la participación activa de estudiantes y familias rurales e intervención de la UNA. Los estudiantes realizaron prácticas pre-profesionales en comunidades rurales interactuaron con las familias, redactaron informes sobre la situación actual, los cuales fueron evaluados a través de escala Likert y estadístico de Cronbach (análisis de evaluación del curso por estudiantes e informes redactados por estudiantes). Posterior a esto, fueron muestreadas encuestas con información de las comunidades rurales de El Callao, Las Pilas 1, Las Pilas 2, Santa Julia y Daniel Téller (municipio de El Crucero, Managua), y relacionadas mediante herramientas estadísticas univariadas y multivariadas.
\end{abstract}

\begin{abstract}
The Rural Integrative Participatory Development program (DEPARTIR) worked with families, students and research teachers from the National Agrarian University, Nicaragua (UNA), the Agricultural University of Natural Resources and Applied Life Sciences (BOKU), Viena and the Casa de los Tres Mundos (CTM) de Granada, in rural communities of Nicaragua with the support of the Austrian Partnership Programme in Higher Education and Research for Development (APPEAR), and The international Treaty on Plant Genetic Resourses for Food and Agriculture (FAO). During the 2006-2015 period, the program had intervention in 17 communities, developed seven diagnostic editions and prioritized proposals jointly with the families. The objetive of this study was to communicate the appreciation and assessment of the theoreticalpractical course developed by DEPARTIR to students and to analyze basic information collected by students in five rural communities in El Crucero, Managua. Surveys were applied in the family production unit (UFP) to find out the socio-economic conditions with the active participation of students and rural families and intervention by UNA. Students carried out pre-professional practices in rural communities, interacted with families, wrote reports on the current situation, which were evaluated through the Likert scale and the Cronbach's Alpha (analysis of course evaluation by students and reports written by students). After this, surveys were sampled with information from the rural communities of El Callao, Las Pilas 1, Las Pilas 2, Santa Julia and Daniel Téller (municipality of El Crucero, Managua), and related using univariate and multivariate statistical tools. The description of population pyramid, a dwelling quality
\end{abstract}

Recibido: 21 de enero del 2020 Aceptado: 11 de mayo del 2020

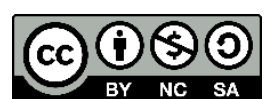

Los artículos de la revista La Calera de la Universidad Nacional Agraria, Nicaragua, se comparten bajo términos de la licencia Creative Commons: Reconocimiento, No Comercial, Compartir Igual. Las autorizaciones adicionales a las aquí delimitadas se pueden obtener en el correo edgardo.jimenez@ci.una.edu.ni

C Copyright 2020. Universidad Nacional Agraria (UNA). 
La descripción de pirámide poblacional, nivel de analfabetismo e índice de calidad de vida de la vivienda caracterizó a estos municipios. Las variables más representativas fueron: área de UFP (X1), personas en el hogar (X2), sexo (X3), edad (X4), tenencia de la tierra (X5), organización (X6), religión (X7), meses de acceso a la carretera (X8) e índice calidad de vida de la vivienda (X9). Este diagnóstico participativo realizado por un grupo interdisciplinario desarrolló competencias en los estudiantes, despertó la cognición al indagar el contexto de las familias rurales; además, esta experiencia fue acreditada como Práctica Pre-profesional en el Plan de Estudios por objetivos. La experiencia e información obtenida a partir de los diagnósticos realizados por DEPARTIR, puede ser retomada y utilizada en la docencia, investigación y extensión para la conformación de proyectos de desarrollo comunitario de carácter interdisciplinario en comunidades rurales de Nicaragua. La población comunitaria es joven, con bajos niveles de analfabetismo, pero con índice de calidad de vida en vivienda moderada. pirámide poblacional es progresiva, Asimismo, la relación de las comunidades estuvo discriminada en un $77 \%$ por las variables X3, X7, X8 y X9. Palabras clave: DEPARTIR, encuestas, Cronbach, análisis univariados y multivariados, índice de calidad de vida en la vivienda, desarrollo comunitario. index characterized these municipalities. The most representative variables were: area of UFP (X1), people in the home (X2), sex (X3), age (X4), land tenure (X5), organization (X6), religion (X7), months access to the highway (X8) and dwelling quality index (X9). This participatory diagnosis carried out by an interdisciplinary group developed competencies in the students, aroused cognition by investigating the context of rural families; Furthermore, this experience was accredited as Pre-Professional Practice in the Curriculum by objectives. The experience and information obtained from the diagnoses carried out by DEPARTIR can be taken up and used in teaching, research and extension for the formation of community development projects of an interdisciplinary nature in rural communities in Nicaragua. The community population is young, with low levels of illiteracy, but with a moderate quality of dwelling quality index. The population pyramid is progressive. Likewise, the relationship of the communities was discriminated in $77 \%$ by the variables $\mathrm{X} 3, \mathrm{X} 7, \mathrm{X} 8$ and $\mathrm{X} 9$.

Keywords: DEPARTIR, surveys, Cronbach's Alpha, univariate and multivariate analyzes, dwelling quality index, community development.

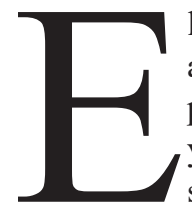

1 trabajo por competencias se considera actualmente como la mejor estrategia formativa para adquirir aprendizajes a lo largo de la vida, ya que son aplicables a una gran diversidad de situaciones (Álvarez, González, y López, 2009). Es por esto, que las prácticas son primordiales dentro de la formación académica, y es una orientación a la realidad profesional; muchos estudiantes llegan a esta etapa de estudio sin conocer lo que van a realizar en la práctica preprofesional (Sevilla, Pila y Guido, 2012).

La Universidad Nacional Agraria (UNA) necesita una mayor integración con la dinámica rural, sobre la base de una construcción conjunta entre la capacidad científicotécnica, dinámica estudiantil, conocimiento y la realidad objetiva de las familias; sin embargo, actualmente la UNA y los centros de difusión agropecuarios, incluyendo las ONGs, no consiguen llegar a toda la población rural y las metodologías utilizadas no generan autonomía ni multiplicación del conocimiento, ya que se aplican métodos preestablecidos sin la intervención de las familias campesinas como sujetos activos que asuman las propuestas (Querol, 2014). El Diagnóstico Rural Participativo (DRP) permite que las comunidades hagan su propio diagnóstico y comiencen a auto-gestionar su planificación y desarrollo (Verdejo, 2003).

Debido a la falta de enseñanza integrada sobre agricultura y relaciones ambientales, un programa educativo puede ser diseñado para que los estudiantes de agronomía y forestales entiendan la complejidad de las interacciones entre las actividades agrícolas, la dinámica del paisaje y la biodiversidad. Asimismo, proponer alternativas de gestión para mejorar la situación actual, de acuerdo con un conjunto de técnicas, en las cuales han sido entrenados previamente (Etienne, Monteil y Legras, 2008). Larke, Hughes, Linhardt, y Weston (1985), mencionan algunas competencias de estudiantes de agronomía, tales como rendimiento e identificación de malezas en cultivos, así como prácticas de labranza, entre otras.

Las prácticas pre-profesionales deben ser evaluadas en las universidades para determinar el nivel de desarrollo de las competencias en el futuro graduado. Las investigaciones sobre esta metodología deben de contribuir con información para la revisión curricular de la universidad, con el objetivo de poder corregir la formación profesional de estudiantes y que éstos sean considerados por los empleadores, productores, propietarios de fincas y que desarrollen temáticas consensuadas con las familias rurales.

Por lo anterior, en el presente estudio se propuso explorar la realidad de las prácticas pre-profesionales que desarrolló el programa de Desarrollo Participativo Integral Rural (DEPARTIR) en cinco comunidades rurales de El Crucero, Managua, Nicaragua, como muestra y modelo de un curso teórico-práctico de Diagnóstico Participativo, teniendo como finalidad la evaluación del curso e informes de Diagnósticos redactados por estudiantes, así como el análisis de muestras de encuestas de la información básica recopilada por los estudiantes y relación de las comunidades rurales de El Crucero mediante herramientas invariadas y multivariadas. 


\section{MATERIALES Y MÉTODOS}

El programa DEPARTIR ofreció propuestas concretas para apoyar el progreso sostenible en áreas rurales. Desde el año 2006 desarrolló VII ediciones de Diagnóstico Participativos en 17 comunidades rurales de Nicaragua en Madriz, Granada, Chinandega y Managua con la participación de familias, estudiantes y docentes investigadores de la UNA, la Universidad Agropecuaria de Viena (BOKU) y la Casa de los Tres Mundos (CTM). La información abarcó estudios de cuestionarios socioeconómicos, suelos, agua y biodiversidad; así como el análisis de la unidad familiar de producción (UFP), entre otros (Cuadro 1).

Metodología del curso teórico-práctico. Organización y planificación de reuniones, asambleas y talleres con los comunitarios antes y después de intervenir a cada una de las comunidades, y posteriormente devolución de resultados y propuestas. Durante el desarrollo de actividades se organizaron los cursos teórico-práctico en seis semanas: capacitación en aula (semana 1), trabajo de campo 1 (semana 2), sistematización de la información (semana 3), trabajo de campo 2 (semana 4), preparación de informes en la UNA y elaboración de propuestas (semana 5 y 6). Durante la estadía en el campo, los estudiantes, un varón y una mujer vivían en el hogar de las familias, por lo que eran dos integrantes más en la familia, y el ama de casa con un pago simbólico era la encargada de alimentar a los estudiantes que posteriormente iban a trabajar en el llenado de encuestas y aplicación de herramientas participativas de diagnósticos en las UFP asignadas. Los docentes se hospedaban en áreas comunales aledañas y ellos mismos preparaban sus alimentos. Todos los días de $5 \mathrm{pm}$ a $7 \mathrm{pm}$, eran realizadas asambleas con estudiantes para hacer la evaluación del trabajo diario. Además, se invitaban comunitarios, si la situación lo ameritaba.

Al finalizar el Curso teórico-práctico, los estudiantes entregaban un Informe a cada familia en donde se reflejaba el diagnóstico realizado por los estudiantes de III y IV año de las diferentes carreras de la UNA, y algunos estudiantes extranjeros. Durante cada período de diagnóstico, los estudiantes estuvieron trabajando en campo, cómputo y en el Centro Nacional de Información y Documentación Agropecuaria (CENIDA), de la UNA, hasta la entrega del informe a las familias por más de 350 horas.

El área de intervención fueron cinco comunidades del municipio de El Crucero ( $11^{\circ} 59^{\prime}$ latitud $\mathrm{N}$ y $11^{\circ} 59^{\prime}$ longitud O, y $860 \mathrm{msnm}$ ), con $210 \mathrm{~km}^{2}$. Limita al Norte con Managua, al Sur con los municipios de San Marcos y San Rafael del Sur; al Este con los Municipios de Ticuantepe y La Concepción. Al Oeste, Limita con el municipio de Villa Carlos Fonseca.
Análisis de informes. La información fue procesada y analizada con SPSS (IBM Corp., 2010) y R (R Core Team, 2019). Fue utilizado el alpha de Cronbach ( $\infty$ de Cronbach) y $\mathrm{T}$ de Students en el análisis de Ítems provenientes de las evaluaciones del curso por parte de estudiantes y evaluación de informes realizadas por docentes especialistas, análisis de correlación paramétrica (Pearson) y no paramétrica (Kendall), comparación de medias mediante Diferencia Mínima Significativa (DMS) y Kruskal-Wallis. Asimismo, se valoró el índice de calidad de vida en la vivienda y tasa de analfabetismo (Agosín y Machado, 2004). La muestra del estudio fue obtenida a través de la ecuación propuesta de Aguilar-Barojas (2005).

$$
n=\frac{N Z^{2} p q}{d^{2}(N-1)+Z^{2} p q}
$$

Con la ecuación anterior se extrajo una muestra de 266 encuestas, en las que se incluyó información de las comunidades de El Callao, Las Pilas 1, Las Pilas 2, Santa Julia y Daniel Téller, del municipio de El Crucero, Managua Cuadro 1.

Relación de comunidades. De las bases de datos con más de mil registros y 100 variables se realizó análisis exploración y discriminatorio y seleccionadas las siguientes variables: área de UFP (X1), personas en el hogar (X2), sexo (X3), edad (X4), tenencia de la tierra (X5), organización (X6), Religión (X7), meses de acceso a la carretera (X8) e índice calidad de vida de la vivienda (X9). Las comunidades fueron relacionadas mediante Análisis de Componentes Principales (ACP), previa transformación a variables dicotómicas $(0,1)$. La transformación de variables doble estado es recomendada por López (2005) en encuestas.

La metodología mixta de análisis es recomendada por Hernández, Fernández, y Baptista (2006), y aplicada por Benavides y Morán (2017); Ruíz (2019).

\section{RESULTADOS Y DISCUSIÓN}

Propuestas de desarrollo comunitario y producción intelectual. El programa DEPARTIR tuvo intervención en comunidades rurales de El Castillito (Madriz), Aquespalapa, El Jicote y Apacunca (Chinandega), El Pegador, Nueva Esperanza y Buena Vista (Madriz), El Callao, Las Pilas 1, Las Pilas 2, Santa Julia, Daniel Téller y Santa Rosa (E1 Crucero, Managua), Los Ángeles, San Mateo, Nandarola y Los Castillos (Granada). 
Cuadro 1. Ítems de encuestas e informes de diagnósticos obtenidos durante la V y VII edición de diagnósticos participativos, El Crucero, Managua

\begin{tabular}{|c|c|c|c|}
\hline Ítems & Evaluación del curso ( $\mathrm{n}=84$ encuestas de estudiantes) & Ítems & $\begin{array}{l}\text { Evaluación de informes ( } \mathrm{n}=110 \text { informes } \\
\text { de diagnóstico) }\end{array}$ \\
\hline \multicolumn{2}{|c|}{ Encuestas } & \multicolumn{2}{|c|}{ Introducción } \\
\hline A1. & ¿Aprendió a hacer encuestas? & al. & Antecedentes \\
\hline A2. & ¿Aprendió a usar nuevos instrumentos de diagnóstico? & a2. & Justificación \\
\hline A3. & ¿Aprendió a hacer un diagnóstico participativo? & a3. & Problemas de estudio \\
\hline \multicolumn{2}{|c|}{ Uso del GPS } & \multicolumn{2}{|c|}{ Análisis de la finca } \\
\hline $\mathrm{B} 1$. & ¿Aprendió a sistematizar datos de campo? & b1. & Uso de los recursos \\
\hline B2. & ¿Aprendió a manejar un GPS? & b2. & Uso del tiempo \\
\hline B3. & ¿Aprendió a hacer levantamientos de campo? & b3. & Consolidación de problemas \\
\hline B4. & ¿Aprendió a limpiar datos de GPS? & & \\
\hline B5. & ¿Aprendió a hacer mapas básicos? & & \\
\hline \multicolumn{2}{|c|}{ Relación con la comunidad y apreciación del curso } & \multicolumn{2}{|c|}{ Componentes socioeconómicos } \\
\hline C1. & $\begin{array}{l}\text { ¿Mejoró su capacidad de integración con la población } \\
\text { campesina? }\end{array}$ & $\mathrm{c} 1$. & Familia \\
\hline $\mathrm{C} 2$. & ¿Entiende ahora mejor la realidad rural? & $\mathrm{c} 2$. & Género \\
\hline $\mathrm{C} 3$. & ¿Este módulo le servirá para su vida profesional? & c3. & Procesos productivos \\
\hline C4. & ¿Mejoró su capacidad de trabajo en equipo? & $\mathrm{c} 4$. & Análisis de recursos de la finca \\
\hline C5. & ¿Cómo valora la participación de los demás estudiantes? & & \\
\hline \multicolumn{2}{|c|}{ Tiempo del curso } & \multicolumn{2}{|c|}{ Descripción de los sistemas productivos } \\
\hline D1. & ¿Se debiera impartir este curso a todos los estudiantes? $*$ & d1. & Finca actual \\
\hline D2. & ¿En qué año de la carrera debiera ser impartido el curso? & $\mathrm{d} 2$. & Procesos productivos \\
\hline D3. & ¿Qué tan bien se aprovecharon las cinco semanas? & $\mathrm{d} 3$. & Otros procesos \\
\hline \multicolumn{2}{|c|}{ Valoración del equipo docente } & \multicolumn{2}{|c|}{ Expectativas } \\
\hline E1. & ¿Cómo valora la participación del profesor principal? & e1. & Finca soñada \\
\hline E2. & ¿Cómo valora la participación de los otros profesores? & $\mathrm{e} 2$. & Finca posible \\
\hline E3. & ¿Cómo valora la participación de los asistentes de curso? & $\mathrm{e} 3$. & Planificación \\
\hline \multicolumn{2}{|c|}{ Valoración del equipo de logística } & \multicolumn{2}{|c|}{ Sobre el informe general } \\
\hline F1. & ¿Cómo valora la participación de los conductores? & fl. & Valoración general del informe \\
\hline $\mathrm{F} 2$. & ¿Cómo valora su propia participación en el curso? & & \\
\hline F3. & ¿Cómo valora la participación de su compañero de equipo? & & \\
\hline
\end{tabular}

Durante el período que subsistió DEPARTIR, fueron muestreadas 865 UFP, realizados 405 diagnósticos y desarrolladas 57 propuestas priorizadas por las familias. Todas estas actividades tuvieron financiamiento por más de 350 mil dólares en todo el período que trabajó DEPARTIR, a esto hay que incluir otros fondos y espacios ofrecidos por UNA.

Entre las propuestas de mayor relevancia se destacaron en orden de frecuencias las siguientes prioridades: Huertos familiares y escolares, producción de granos básicos y hortalizas, fertilizantes orgánicos, microtúneles para plántulas, producción orgánica de café, manejo de frutales y ornamentales, perforación de pozos, aves de patio, alimentación de ganado mayor $\mathrm{y}$ menor en época seca, cosecha de agua, control de plagas y enfermedades en cultivos y animales, agroindustria de productos, conservación del teocintle (Zea nicaraguensis
Iltis \& Benz), entre otros. Algunas propuestas desarrolladas por grupos pequeños de productores fueron financiadas por DEPARTIR.

Las bases de datos están conformadas con información de 4037 personas de las diferentes edades. En dichos diagnósticos participaron 193 estudiantes y obtenido su certificado (convalidado como prácticas preprofesionales) y más de 30 docentes, entre nacionales y extranjeros.

En cuanto a divulgación y producción intelectual, se destacan tres tesis de posgrado nacionales y dos extranjeras, y más de 20 tesis de grado y graduados más de 30 estudiantes, 15 artículos científicos, 13 videos en el programa televisivo en la Tierra de Xilonen, siete informes de diagnósticos, más de seis guías y un libro que plasma la experiencia DEPARTIR. Además de participar en jornadas científicas y congresos nacionales e internacionales. 
Evaluación del curso e informes. Los estudiantes evaluaron el curso como instrumento en las prácticas preprofesional en el campo, y en general los ítems tuvieron Buena fiabilidad con valores promedios de 0.66 y 0.80 para los diagnósticos muestreados, y promedios de 0.73 (Cuadro 2). Cervantes (2005), afirma que el coeficiente de Cronbach estima consistencia interna del conjunto de ítems, evalúa la magnitud y correlación de instrumentos. Un valor superior a 0.70 es consistencia buena; pero también son aceptables valores mayores de 0.6 en investigación exploratoria (Oviedo y Campo, 2005).
Las diferentes agrupaciones de ítems resultaron altamente significativas, y algunos ítems individuales (A-F) variaron según la apreciación de los estudiantes. Los estudiantes marcaron como bastante (4) aceptación el curso que desarrolló DEPARTIR en las comunidades rurales. En muchos casos, la marcación de las preguntas fue de muchísima (5) aceptación del curso por parte de los estudiantes (Cuadro 2).

La escala Likert agrupó afirmaciones desfavorables (grupo inferior) y favorables (grupo superior), y correspondieron a las puntuaciones $1-2$ y $4-5$, respectivamente

Cuadro 2. Ítems y parámetros estadísticos en la evaluación del curso y evaluación de informes redactados por los estudiantes. Municipio de El Crucero, Managua

\begin{tabular}{|c|c|c|c|c|c|c|c|c|c|c|c|}
\hline \multirow[t]{2}{*}{ Items } & \multirow[t]{2}{*}{$\infty$} & Muestra I & \multicolumn{3}{|c|}{ Muestra II } & \multirow[t]{2}{*}{ Ítems } & \multicolumn{2}{|r|}{ Muestra I } & \multicolumn{3}{|c|}{ Muestra II } \\
\hline & & Inf-Sup & $\infty$ & Inf-Sup & $\bar{Y}$ & & $\infty$ & Inf-Sup & $\infty$ & Inf-Sup & $\bar{Y}$ \\
\hline \multicolumn{6}{|c|}{ Encuestas } & \multicolumn{6}{|c|}{ Introducción y metodología } \\
\hline A1 & 0.65 & $4.14-4.71^{\mathrm{NS}}$ & 0.82 & $4.38-4.23^{\mathrm{NS}}$ & $4.31^{\mathrm{NS}}$ & al & 0.84 & $4.95-4.96^{\mathrm{NS}}$ & 0.84 & $5.00-4.96^{\mathrm{NS}}$ & $4.95^{\mathrm{NS}}$ \\
\hline $\mathrm{A} 2$ & 0.64 & $3.86-4.48^{\mathrm{NS}}$ & 0.87 & $4.15-4.69^{\mathrm{NS}}$ & & a2 & 0.84 & $4.85-4.86^{\mathrm{NS}}$ & 0.84 & $4.85-4.86^{\mathrm{NS}}$ & \\
\hline \multirow[t]{2}{*}{ A3 } & 0.69 & $4.10-4.00^{\mathrm{NS}}$ & 0.79 & $4.31-4.62^{\mathrm{NS}}$ & & $\mathrm{a} 3$ & 0.85 & $4.97-4.98^{\mathrm{NS}}$ & 0.85 & $4.97-4.98^{\mathrm{NS}}$ & \\
\hline & & $4.23 * *$ & & $4.95 * *$ & & & & $4.21^{\mathrm{NS}}$ & & $4.95^{\mathrm{NS}}$ & \\
\hline \multicolumn{6}{|c|}{ Uso del GPS } & \multicolumn{6}{|c|}{ Análisis de la UFP } \\
\hline $\mathrm{B} 1$ & 0.64 & $3.62-4.24 * *$ & 0.81 & $4.23-4.46^{\mathrm{NS}}$ & $4.04^{\mathrm{NS}}$ & b1 & 0.84 & $1.41-2.23^{\mathrm{NS}}$ & 0.71 & $1.58-2.62 * *$ & $1.87^{\mathrm{NS}}$ \\
\hline $\mathrm{B} 2$ & 0.66 & $3.95-4.48 * *$ & 0.80 & $3.77-4.46^{* *}$ & & b2 & 0.85 & $1.41-1.62^{\mathrm{NS}}$ & 0.70 & $1.67-2.31 * *$ & \\
\hline B3 & 0.65 & $3.71-4.14^{\mathrm{NS}}$ & 0.80 & $3.92-4.62 * *$ & & b3 & 0.84 & $1.38-2.00^{\mathrm{NS}}$ & 0.64 & $1.50-2.54 * *$ & \\
\hline B4 & 0.67 & $3.43-4.05^{\mathrm{NS}}$ & 0.79 & $3.31-4.38 *$ & & & & $1.68 * *$ & & $2.06 * *$ & \\
\hline B5 & 0.67 & $\begin{array}{c}3.71-4.05^{\mathrm{NS}} \\
3.93 * *\end{array}$ & 0.80 & $\begin{array}{c}3.92-4.31^{\mathrm{NS}} \\
4.14^{* *}\end{array}$ & & & & & & & \\
\hline \multicolumn{6}{|c|}{ Relación con la comunidad } & \multicolumn{6}{|c|}{ Componentes socioeconómicos } \\
\hline $\mathrm{C} 1$ & 0.65 & $3.62-4.24 * *$ & 0.80 & $4.38-4.54^{\mathrm{NS}}$ & $4.78^{\mathrm{NS}}$ & $\mathrm{c} 1$ & 0.82 & $1.08-2.15^{* *}$ & 0.68 & $1.50-2.69 * *$ & $1.83 * *$ \\
\hline $\mathrm{C} 2$ & 0.68 & $3.95-4.48^{\mathrm{NS}}$ & 0.79 & $4.54-4.62^{\mathrm{NS}}$ & & $\mathrm{c} 2$ & 0.82 & $1.00-1.85 * *$ & 0.69 & $1.58-2.54 * *$ & \\
\hline $\mathrm{C} 3$ & 0.64 & $3.71-4.14 * *$ & 0.80 & $4.54-4.77^{\mathrm{NS}}$ & & c3 & 0.83 & $1.08-1.85^{* *}$ & 0.69 & $1.75-2.62 * *$ & \\
\hline $\mathrm{C} 4$ & 0.63 & $3.43-4.05 * *$ & 0.80 & $3.62-3.92^{\mathrm{NS}}$ & & $\mathrm{c} 4$ & 0.82 & $1.17-1.77 * *$ & 0.71 & $1.50-2.54 * *$ & \\
\hline $\mathrm{C} 5$ & 0.67 & $\begin{array}{c}3.71-4.05^{\mathrm{NS}} \\
4.28 * *\end{array}$ & 0.78 & $\begin{array}{c}4.00-4.77 * * \\
4.37 * *\end{array}$ & & & & $1.51 * *$ & & $2.13 * *$ & \\
\hline \multicolumn{6}{|c|}{ Tiempo del Curso } & \multicolumn{6}{|c|}{$\begin{array}{l}\text { Descripción de los sistemas } \\
\text { productivos }\end{array}$} \\
\hline D1 & 0.68 & $4.24-4.48^{\mathrm{NS}}$ & 0.82 & $5.00-5.00^{\mathrm{NS}}$ & $3.14 *$ & d1 & 0.86 & $2.25-2.00^{\mathrm{NS}}$ & 0.67 & $1.67-2.62 * *$ & $1.87^{\mathrm{NS}}$ \\
\hline D2 & 0.69 & $3.67-4.14^{\mathrm{NS}}$ & 0.81 & $3.15-3.46^{\mathrm{NS}}$ & & $\mathrm{d} 2$ & 0.82 & $1.00-2.08 * *$ & 0.72 & $1.50-2.46^{* *}$ & \\
\hline D3 & 0.65 & $\begin{array}{c}2.62-3.48 * * \\
2.72 * *\end{array}$ & 0.79 & $\begin{array}{c}4.15-4.54^{\mathrm{NS}} \\
2.89^{* *}\end{array}$ & & $\mathrm{~d} 3$ & 0.83 & $\begin{array}{c}1.08-1.69 * * \\
1.68 * *\end{array}$ & 0.72 & $\begin{array}{c}1.58-2.46^{* *} \\
2.06^{*}\end{array}$ & \\
\hline \multicolumn{6}{|c|}{$\begin{array}{l}\text { Valoración del equipo } \\
\text { docente }\end{array}$} & \multicolumn{6}{|c|}{ Expectativas de la familia } \\
\hline E1 & 0.66 & $4.24-4.48^{\mathrm{NS}}$ & 0.81 & $4.00-3.85^{\mathrm{NS}}$ & $4.01 *$ & e1 & 0.82 & $1.00-1.84 * *$ & 0.62 & $1.58-3.08 * *$ & $1.79 *$ \\
\hline E2 & 0.65 & $3.67-4.14^{* *}$ & 0.79 & $4.08-4.93 * *$ & & $\mathrm{e} 2$ & 0.82 & $1.00-2.00 * *$ & 0.62 & $1.50-2.69 * *$ & \\
\hline E3 & 0.66 & $\begin{array}{c}2.62-3.48 * * \\
3.78 * *\end{array}$ & 0.79 & $\begin{array}{c}4.00-4.62 * * \\
4.24 * *\end{array}$ & & e3 & 0.82 & $\begin{array}{c}1.00-1.69 * * \\
1.447 * *\end{array}$ & 0.62 & $\begin{array}{c}1.42-2.46^{*} \\
2.14^{* *}\end{array}$ & \\
\hline \multicolumn{6}{|c|}{$\begin{array}{l}\text { Valoración general del } \\
\text { informe }\end{array}$} & \multicolumn{6}{|c|}{ Valoración general del informe } \\
\hline F1 & 0.66 & $3.10-4.05 * *$ & 0.79 & $4.00-3.85 * *$ & $4.21 *$ & fl & 0.80 & $1.96-2.97 * *$ & 0.75 & $2.22-3.04 * *$ & $2.44^{*}$ \\
\hline $\mathrm{F} 2$ & 0.65 & $3.90-4.43 * *$ & 0.78 & $4.08-4.93 * *$ & & & & & & & \\
\hline F3 & 0.65 & $\begin{array}{c}3.90-4.33 \mathrm{NS} \\
3.87 * *\end{array}$ & 0.79 & $\begin{array}{c}4.00-4.62 * * \\
4.50^{* *}\end{array}$ & & & & & & & \\
\hline
\end{tabular}

$\mathrm{Inf}=$ media grupo inferior, Sup=media grupo superior. $\overline{\mathrm{Y}}=$ media. $\mathrm{NS} p>0.05,{ }^{*} p<0.05,{ }^{* *} p<0.01 . \infty$ (Alpha de Cronbach) y T. 
(Hernández, Espejo, González, y Gómez, 2001; López, 2005; Guil, 2006). Mediante $t$ de Student se discriminó la consistencia interna de los resultados (Elejabarrieta e Iñiguez, 1984; Blanco y Alvarado, 2005).

El curso teórico-práctico que impartió DEPARTIR determinó en los estudiantes capacidades aplicadas de entendimiento, análisis y toma de decisiones ante las distintas realidades rurales, a través de enfoques y metodologías participativas, para examinar la situación de una comunidad rural

La evaluación de informes redactados por estudiantes (a-f) presentaron valores promedios de $\infty=0.83$ y $\infty=0.75$ para las Muestras I y II, respectivamente (Cuadro 2). Aunque la consistencia de los ítems fue Buena, los valores promedios en la escala Likert presentaron categorías de informes con una valoración promedio general significativo e inclinación al valor de Regular $\left(2.23^{* *}\right.$ y $\left.2.64 * *\right)$. Estos resultados pudieron ser debidos a la complejidad de evaluación de los componentes de las UFP (b1-b3, c1-c4) por parte de los estudiantes que aún no han culminado su Plan de Estudio.

Los estudiantes de la UNA con su participación activa en los diagnósticos participativo redactaron y sistematizaron Informes de las UFP en el Centro de Cómputo de la UNA para entregar a las familias en las comunidades en conjunto con el equipo interdisciplinario de DEPARTIR. Los estudiantes conocieron la situación actual de las familias y les permitió identificar y valorar la problemática en la comunidad rural.

Las prácticas, constituyen un componente esencial de la formación de los estudiantes de educación superior, quienes con ellas inician su inserción en el mundo laboral por un período determinado, tendiéndose así un puente entre la teoría y la práctica, entre la etapa formativa y el ingreso al mercado de trabajo (De la Vega y Arakaki, 2011).

Kulalova (2013), considera que una de las valiosas e importantes experiencias para los estudiantes universitarios, desde el punto de vista de su formación profesional, son las prácticas profesionales; estas a su vez es un tema de constante análisis, preocupación y discusión, por la relevancia que tiene esta instancia en los procesos de enseñanza-aprendizaje (Roza y Touzé, 2011).
Información básica obtenida en las UFP. La información de mayor importancia recopilada por los estudiantes fue objeto de análisis para relacionar las cinco comunidades rurales de El municipio de El Crucero. En este caso, se seleccionaron datos de los integrantes de las familias y el nivel de analfabetismo.

Pirámide poblacional y analfabetismo. Las muestras de las comunidades fueron agrupadas en tres categorías: el $22.6 \%$ estuvo representada por jóvenes menores a 14 años $(11.9 \%$ hombres, $10.7 \%$ mujeres), $71.8 \%$ adultos entre 15 y 64 años (37.3\% hombres, $34.5 \%$ mujeres), y personas de la tercera edad superior a los 65 años (3.6\% hombres, $2.1 \%$ mujeres). La media fue de 30.26 años y una desviación típica de 18.65

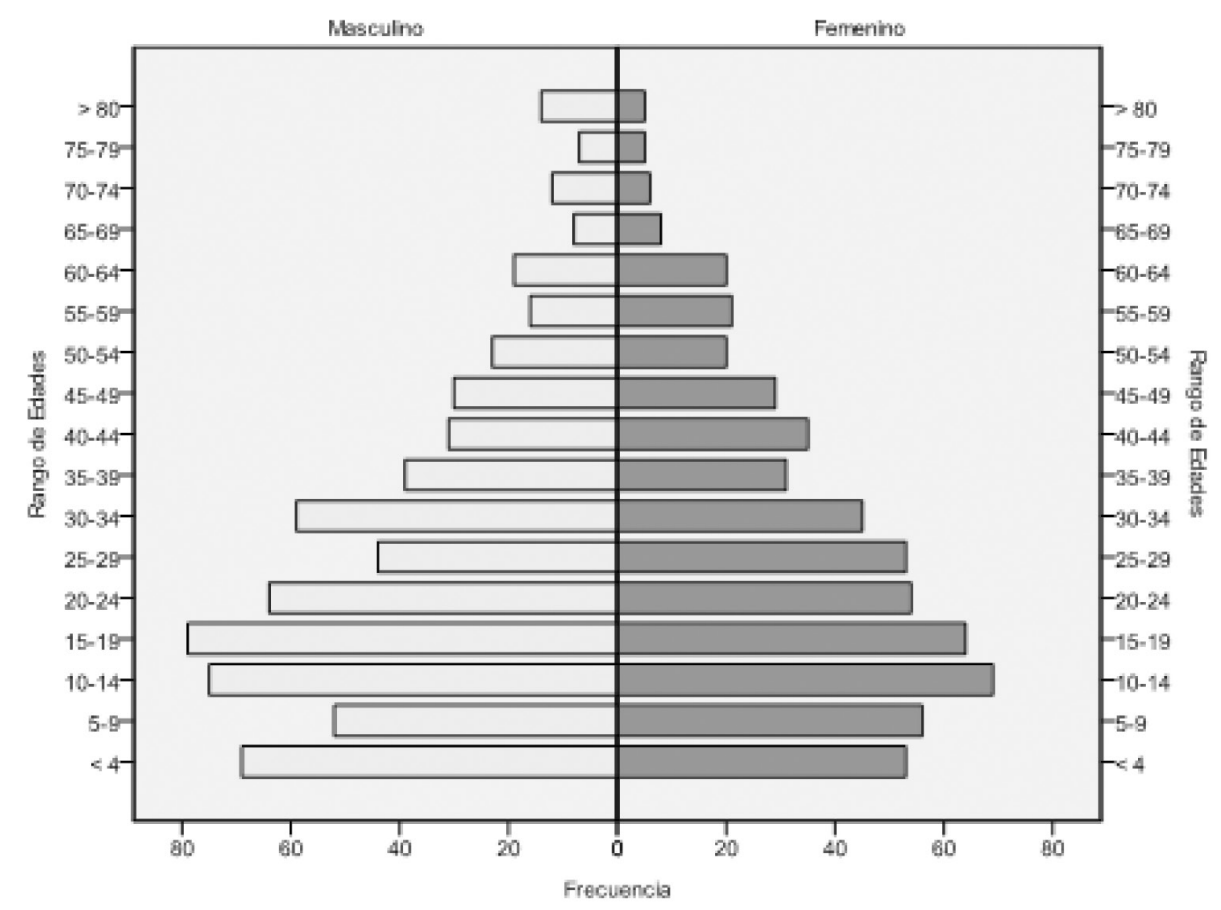

Figura 1. Pirámide poblacional según el sexo en cinco comunidades rurales muestreada en El Crucero, Managua. $\mathrm{n}=1214$. años, lo que indica una población relativamente joven. Los porcentajes de hombres en Daniel Téller, El Callao, Las Pilas 1, Las Pilas 2 y Santa Julia fueron de 51.4\%, 50.2\%, 52.5\%, $53.5 \%, 54.7 \%$ y $52.7 \%$, respectivamente. El engrosamiento de la pirámide progresiva (Figura 1), es propio de una población en aumento y con alta tasa de crecimiento, en la que sobresalen los rangos de edades entre 0-24 años. El 76.3\% de las personas mostraron edades inferiores a los 40 años.

En las comunidades estudiadas (Cuadro 3), el total de analfabetos mayores de 15 años fue del 52\% (hombres) y 48\% (mujeres). Las comunidades de Las Pilas 2 y Las Pilas 1 , presentaron los mayores porcentajes de analfabetismo, con $4.0 \%$ y 4.64 respectivamente. 
La tasa promedio general de analfabetismo en personas mayores de 15 años fue de $2.9 \%$. Se observó que las tasas medias de analfabetismo fueron altamente significativas $(p<0.001)$, y mayores a medida que la edad incrementaba (Pearson $=0.81, p=0.001$ ). El analfabetismo se intensificó en las personas entre 55-64 años (Cuadro 3); asimismo el analfabetismo con los rangos de edades presentó correspondencia significativa (Kendall $=0.90, \quad p=0.001$ ). Benavides y Morán (2013); y Ruíz (2019), reportan resultados muy similares en comunidades rurales. factor crítico para financiar la infraestructura física y social requerida y, a la vez, combatir los altos niveles de pobreza que todavía afectan a cerca del $40 \%$ de la población.

Según el Instituto International de Planeamiento de la Educación / United Nations Educational, Scientific and Cultural Organization (IIPE/UNESCO, 2007), el analfabetismo en los países centroamericanos ha persistido a través de los años, a pesar de las diversas políticas educativas y planes de acción que se han instaurado en pro de su eliminación. Durante la década de los noventa el 27\% de la población centroamericana con más de 15 años sonanalfabetas.
Cuadro 3. Analfabetismo en rangos de edades y significación estadística del promedio en cinco comunidades rurales de Nicaragua

\begin{tabular}{lccccccccccc}
\hline Comunidad & $15-24$ & $25-34$ & $35-44$ & $45-54$ & $55-64$ & $25-64$ & $>15$ años & & \multicolumn{2}{c}{ Medias } \\
\hline Las Pilas 1 & 1.9 & 3.0 & 2.3 & 6.9 & 11.1 & 4.8 & 5.2 & & 4.64 & $\mathrm{a}$ \\
Las Pilas 2 & 0.4 & 1.0 & 5.3 & 3.9 & 12.5 & 4.4 & 4.1 & & 4.00 & $\mathrm{ab}$ \\
El Callao & 0.4 & 1.0 & 5.3 & 2.9 & 6.9 & 3.4 & 2.7 & & 2.88 & $\mathrm{abc}$ \\
Santa Julia & 0.0 & 1.0 & 0.8 & 2.9 & 5.6 & 2.0 & 1.7 & & 1.75 & $\mathrm{bc}$ \\
Daniel Téller & 0.0 & 0.0 & 0.0 & 1.0 & 4.2 & 0.8 & 0.6 & & 0.83 & $\mathrm{c}$ \\
\hline
\end{tabular}

Medias en común no difieren estadísticamente según DMS $(p>0.05)$.

La educación es determinante para potenciar el desarrollo integral de un país, por lo que el capital humano acumulado, permite mejorar las condiciones de vida de la población. El mayor nivel de educación y formación se traducen en mayores y mejores oportunidades de empleo y condiciones de vida según el Instituto Nacional de Información de Desarrollo (INIDE, 2007).

El analfabetismo resulta de la interacción de una serie de factores, entre los cuales destacan: la pobreza, la desnutrición, los problemas de salud, el trabajo infantil, la migración y la falta de acceso a entornos de enseñanza y aprendizaje en forma continua. Estas desigualdades inciden en la vulnerabilidad social de las personas y varían, aún más, según género, edad, etnia y contexto geográfico (Martínez, Trucco, y Palma, 2014). La Universidad Centroamericana (UCA, 2010), menciona que los pobres extremos no alcanzan un buen nivel educativo, ya que el $41 \%$ no tiene ningún nivel de instrucción y el 50.6\% únicamente logra cursar la primaria. Lestage (1982), asevera que existe una relación del analfabetismo con la demografía, y la relación es cuantitativa y cualitativa, y asevera que el analfabetismo es inherente al grado de subdesarrollo de un país.

Según Agosín y Machado (2004), los estados centroamericanos sólo recaudan el $13.5 \%$ del producto interno bruto en ingresos tributarios. La falta de recursos resultante hace que el gasto público sea insuficiente y de baja calidad y genere déficits fiscales crónicos que son financiados mediante endeudamiento. En el 2003 los intereses alcanzaron, en promedio, al 18\% de los ingresos impositivos. En estas economías abiertas que necesitan afianzar la competitividad internacional de sus empresas, la política fiscal se vuelve el
Calidad de vida de la vivienda (ICVV). El ICVV (X9) integra las siguientes dimensiones: Condiciones sanitarias (CS), Condiciones físicas de la vivienda (CFV), Ambiente de la vivienda (AV), Servicios básicos (SB). Dicho índice corresponde a la sumatoria $0.3(\mathrm{CS}), 0.3(\mathrm{CFV}), 0.2(\mathrm{AV}) \mathrm{y}$ $0.2(\mathrm{SB})$. El valor de 0 y 10 corresponde a la peor y mejor condición de ICVV, respectivamente. El índice abarca principios básicos del análisis multicriterio, selecciona las características que tiene una vivienda para garantizar bienestar en la población y la clasificación va de calidad muy alta (>8.1), alta (6.1-8), moderada (4.1-6), baja (2.1-4) y muy baja calidad (Salas-Borgoin, 2012).

En las cinco comunidades muestreadas (Cuadro 3), el $55.2 \%$ de las familias tuvieron el servicio de eliminación de excretas (Cuadro 4). Estos datos no contrastan con la media nacional expuesta por Fundación Internacional para el Desafío Económico Global (FIDEG, 2009), en la que asevera que sólo el $22.1 \%$ de las comunidades rurales tienen este servicio inadecuado. Los materiales más utilizados en las paredes fueron: madera (43.6\%), adobe $(21.3 \%)$, bloque (18.1), ladrillo (8\%), cantera $(2.6 \%)$, zinc $(2 \%)$, bambú $(1.6 \%)$, cartón $(0.2 \%)$, entre otros. Según Fundación Internacional para el Desafío Económico Global (FIDEG, 2009), la proporción de hogares con materiales en la vivienda inadecuados en la población rural es del $64 \%$ en piso, $6.3 \%$ en paredes y $3.6 \%$ en techo.

El abastecimiento de agua fue del $59.6 \%$, y un $39.4 \%$ no contó con el servicio de agua potable en las cinco comunidades estudiadas. El 39\% de la población nicaragüense del área rural cuenta con este servicio ya sea por medio de acueductos, comité de agua potable en zonas rurales, y puestos públicos, indica el Instituto Nacional de Estadísticas y Censos (INEC, 1995). En Nicaragua, 67\% de la población rural está en la pobreza; y de esto el $49.6 \%$ de las familias están en la pobreza no extrema, y $18.2 \%$ se ubica en pobreza extrema Fundación Internacional para el Desafío Económico Global (FIDEG, 2009), además de no contar con energía eléctrica Universidad Centroamericana (UCA, 2010). El 89\% en los ejes de inercia indicaron una considerable explicación de la variación $(\infty=0.90)$. Con el $48 \%$ de varianza se agrupó a las 
comunidades de El Callao, Las Pilas 1 y Las Pilas 2, con la valoración de ICVV de 4-6, lo que indica una moderada calidad de vida en la vivienda (Cuadro 4). Salas-Borgoin, (2012), reporta un valor medio de 5.14 de ICVV, lo que es muy similar al encontrado en el presente estudio.

Cuadro 4. Significación estadística en las variables de condiciones de vida en la vivienda en cinco comunidades de rurales de Nicaragua

\begin{tabular}{|c|c|c|c|c|c|c|c|c|c|c|}
\hline Comunidad & $\mathrm{CS}$ & & CFV & & $\mathrm{AV}$ & & SB & & \multicolumn{2}{|l|}{ ICVV } \\
\hline Las Pilas 2 & 6.33 & $\mathrm{a}$ & 6.42 & $\mathrm{a}$ & 2.47 & $\mathrm{~b}$ & 8.53 & $\mathrm{a}$ & 5.94 & $\mathrm{a}$ \\
\hline Las Pilas 1 & 4.75 & $\mathrm{~b}$ & 6.17 & $a b$ & 3.16 & $\mathrm{a}$ & 7.35 & $\mathrm{a}$ & 5.36 & $\mathrm{a}$ \\
\hline El Callao & 5.02 & $a b$ & 5.89 & $a b$ & 3.96 & $\mathrm{a}$ & 5.60 & b & 5.12 & $a b$ \\
\hline Daniel Téller & 6.33 & $\mathrm{a}$ & 4.65 & $\mathrm{~b}$ & 1.40 & $\mathrm{c}$ & 4.13 & $a b$ & 4.13 & $b$ \\
\hline Santa Julia & 6.73 & $\mathrm{a}$ & 6.33 & $\mathrm{a}$ & 3.22 & $\mathrm{a}$ & 4.74 & $\mathrm{~b}$ & 5.26 & $\mathrm{ab}$ \\
\hline$\overline{\mathrm{Y}}$ & 5.83 & & 5.89 & & 2.84 & & 6.07 & & 5.16 & \\
\hline$p$ & 0.0001 & & $<0.0001$ & & $<0.0001$ & & $<0.0001$ & & $<0.001$ & \\
\hline
\end{tabular}

Promedios con letra (s) en común (columnas), no difieren estadísticamente ( $p>0.05)$ según test de Kruskal-Wallis.

Análisis de componentes principales (ACP). Al integrar el ICVV (X9) con las variables discriminantes y realizar el ACP, los dos primeros componentes principales (CP) extrajeron el $87 \%$ de la desviación total con un Coeficiente Cofenético (CC) muy alto para establecer aseveración de la relación encontrada. El grado de correlación de las variables con respecto a los componentes principales fue inherente al aporte de las variancias a los mismos (Cuadro 5). Los objetivos del ACP, es generar variables nuevas que expresen la información original, reducir la dimensión, y descartar variables correlacionadas y de menor inferencia en la variación (Cuadras, 2007).

Cuadro 5. Correlación y aporte de las variables en los tres primeros componentes principales

\begin{tabular}{|c|c|c|c|c|c|c|}
\hline \multirow[t]{2}{*}{ Variable } & \multicolumn{3}{|c|}{ Correlación (r) } & \multicolumn{3}{|c|}{ Aporte (\%) } \\
\hline & CP 1 & CP 2 & CP 3 & CP 1 & CP 2 & CP 3 \\
\hline $\mathrm{X} 1$ & 0.45 & 0.78 & $-0,33$ & 4.84 & 17.64 & 13.69 \\
\hline $\mathrm{X} 2$ & 0.43 & 0.88 & 0.14 & 4.41 & 22.09 & 2.56 \\
\hline X3 & 0.99 & 0.01 & -0.06 & 23.04 & 0.01 & 0.49 \\
\hline $\mathrm{X} 4$ & 0.56 & 0.32 & 0.76 & 7.29 & 2.89 & 72.25 \\
\hline X5 & -0.55 & 0.78 & -0.08 & 6.76 & 16.81 & 0.81 \\
\hline X6 & 0.11 & 0.95 & -0.07 & 0.25 & 25.00 & 0.64 \\
\hline X7 & 0.89 & 0.35 & 0.06 & 18.49 & 3.61 & 0.49 \\
\hline X8 & 0.87 & 0.49 & 0.07 & 17.64 & 6.76 & 0.64 \\
\hline X9 & 0.87 & 0.41 & 0.27 & 17.64 & 4.84 & 9.61 \\
\hline
\end{tabular}

Según el ACP, el eigenvector 1 ( $\lambda 1)$ discriminó al sexo (X3), la religión (X7), acceso a la carretera (X8) y el índice de calidad de vida en la vivienda (X9) como las variables que relacionaron a las comunidades con un $76.81 \%$ de desviación en el CP-1, y congregaron las comunidades de Daniel Téller, Las Pilas 1 Las Pilas 2. La comunidad de Santa Julia se relacionó con Daniel Téller por poseer niveles similares de X9. Por otro lado, X6, X2 y X1 separaron a las comunidades con un $64.73 \%$ en el CP-2. Las variables restantes no ayudaron a discriminar a las comunidades.

Mediante las actividades realizadas por DEPARTIR, se reflexiona que la información recopilada a partir de la intervención en las comunidades rurales de Nicaragua por parte de la UNA es insumo para desarrollar de manera interdisciplinaria y holística estrategias de desarrollo comunitario, considerando los Objetivos de Desarrollo Sostenible, además de incorporar los resultados a las actividades de la Academia, Investigación y Extensión, así como a la Producción Intelectual.

\section{CONCLUSIONES}

En el curso teórico-práctico desarrollado por DEPARTIR y convalidado como prácticas pre-profesionales en su momento, los estudiantes desarrollaron competencias y habilidades cognitivas sobre el ambiente y la sociedad, conocieron la situación y convivencia familiar; por lo que estas experiencias son relevantes para su formación profesional, social y humana. No obstante, durante las capacitaciones e intercambio se pudo constatar que los estudiantes ameritan reforzar en su plan de estudios las áreas disciplinarias afines a la Redacción Técnica y Matemáticas.

Los integrantes de las familias en las comunidades muestreadas obedecen a una pirámide progresiva, lo que demuestra una población relativamente joven con promedio de 30 años de edad. El porcentaje de analfabetismo no superó el 5\%, y el índice de calidad de vida en la vivienda es moderado. Según el análisis de componentes principales; el sexo, la religión, el acceso a la carretera e índice de calidad de vida en la vivienda ayudaron a asociar con mayor precisión a las comunidades rurales de El Crucero.

\section{AGRADECIMIENTOS}

A las familias de las comunidades rurales de El Callao, Las Pilas 1, Las Pilas 2, Santa Julia y Daniel Téller, por la información brindada, así como por la atención prestada a los estudiantes. A los estudiantes participantes de la UNA, nacionales y extranjeros. Al equipo DEPARTIR (Glenda Martínez Rugama, José Cisne Contreras, Daniel Querol, Juan Carlos Morán Centeno, Félix Nieto, Álvaro Benavides González). Al Programa Austríaco de EducaciónSuperioreInvestigaciónparaelDesarrollo(APPEAR), Organización de las Naciones Unidas para la Alimentación y la Agricultura, Universidad Nacional Agraria (UNA) por el apoyo financiero; así como Universidad Agropecuaria de Viena (BOKU) y Casa de los Tres Mundos (CTM), compañeros y acompañantes en el proyecto: Cambiando Mentes y Estructuras: El creciente involucramiento de la UNA con las comunidades rurales. A las Autoridades superiores de la UNA: Ing. Telémaco Talavera y Dr. Dennis Salazar Centeno, por el apoyo y logística brindada durante y después del desarrollo de los Diagnósticos Participativos en las comunidades rurales de Nicaragua. 


\section{REFERENCIAS BIBLIOGRÁFICAS}

Agosín, M. y Machado, R. (2004). Reforma tributaria y desarrollo humano en Centroamérica. Recaudar Para Crecer, 1.

Aguilar-Barojas, S. (2005). Fórmulas para el cálculo de la muestra en investigaciones de salud. Salud en Tabasco, 11(1-2),333338.

Álvarez, P., González, A., y López, A. (2009). La enseñanza universitaria y la formación para el trabajo. Un análisis desde la opinión de los estudiantes. PARADIGMA, (XXX)2, 7-19.

Benavides, Á., y Morán, J. (2013). Análisis numérico de características básicas de Unidades Familiares Productivas (UFP) en nueve comunidades rurales de Nicaragua. La Calera, 13(21), 101-109.

Benavides, Á. y Moran, J. (2017). Caracterización numérica de la diversidad forestal de 14 de comunidades rurales en cinco municipios de Nicaragua. La Calera, 17(29), 68-77.

Blanco N., y Alvarado, E. (2005). Escala de actitud hacia el proceso de investigación social. Revista de Ciencias Sociales (Ve). $\mathrm{XI}(3)$, 537-544. www.redalyc.org.

Cervantes, V. H. (2005). Interpretaciones del coeficiente alpha de Cronbach. Avances en Medición. Universidad Nacional de Colombia, Bogotá, Colombia. 3, 9-28.

Cuadras, C. M. (2007). Nuevos métodos de análisis multivariante. Barcelona: CMC editions.

De la Vega, A., y Arakaki, M. (2011). Las prácticas pre-profesionales en la formación en ciencias de la información: el caso de la Pontificia Universidad Católica del Perú (PUCP). Revista Interamericana de Bibliotecología, 34(1), 77-86.

Elejabarrieta F. J., e Iñiguez, L. (1984). Construcción de escalas de actitud tipo Thurst y Likert. http:/www.ict.edu.mx/acervo/_ bibliotecologia_escalas-escalas\%20Likert-Thust.pdf.

Etienne, M., Monteil, C., y Legras, M. (2008). Teaching companion modelling to agronomy students: An original way to integrate concepts in agriculture and environment. In Proceedings of the 8th European IFSA Symposium. pp. 979-983.

Fundación Internacional para el Desafío Económico Global. (2009). Encuesta de hogares para la medición de la Pobreza en Nicaragua. 30 p.

Guil, B. (2006). Escala mixta Likert-Thurstone. Anduli. (5), 81-95. http:/institucional.us.es/revistas/anduli/5/art_6.pdf.

Hernández, B., Espejo, T., González, R., y Gómez, B. (2001). Escalas de respuesta tipo Likert: ¿Es relevante la alternativa "Indiferente"?. Metodología de encuestas. 3(2), 135-150.

Hernández Sampieri, R., Fernández Collado, C., y Baptista Lucio, P. (2006). Metodología de la investigación (Cuarta edición.). México D.F.: McGraw-Hill Interamericana.

IBM Corp. Released. (2010). IBM SPSS Statistics for Windows, Version 19.0. Armonk, NY: IBM Corp.

Instituto International de Planeamiento de la Educación / United Nations Educational, Scientific and Cultural Organization. (2007) “Educación para todos en 2015: ¿alcanzaremos la meta?”. Síntesis con énfasis en América Latina y Argentina. Buenos Aires, Argentina.

Instituto Nacional de Información de Desarrollo. (2007). Nicaragua: Estimaciones y Proyecciones de Población Nacional. 19502050. Revisión 2007. 39 p.

Instituto Nacional de Estadísticas y Censos. (1995). VII Censo de Población y III de Vivienda. http://www.inide.gob.ni/censo95/ censo95.htm

Kulalova, O. V. (2013). Prácticas profesionales, una contribución a la mejora del curriculum en la carrra de psicología de la UNAN-León. Universitas, 4(1), 29-32.

Larke, J, A., Hughes, J., Linhardt, R., y Weston, R. (1985). Competencies of agronomy graduates. NACTA Journal. pp. 75-77.

Lestage, A. (1982). Analfabetismo y alfabetización. Organización de las Naciones Unidas para la Educación, La Ciencia y la Cultura (UNESCO). 32 p.

López, P. J. (2005). Ítems politómicos vs. dicotómicos: Un estudio metodológico. Anales de psicología. Universidad de Murcia, España. 21(2), 339-334.

Martínez, R., Trucco, D., y Palma, A. (2014). El analfabetismo funcional en América Latina y el Caribe: Panorama y principales desafíos de política.

Oviedo, C., y Campo, A. (2005). Aproximación al uso del uso del coeficiente alpha de Cronbach. Revista Colombiana de Psiquiatría. (XXXIV)4, 572-580. www.redalyc.org.

Querol, L., Benavides, G., Cisne, C., Morán, J., Nieto, F, Schauppenlener, T., y Yepes (2014). Cambiando mentes y estructuras: manual del Curso Diagnóstico Participativo Integral Rural. Universidad Nacional Agraria. Managua, Nicaragua. 200 p.

R Core Team. (2019). R: A language and environment for statistical computing. R foundation for statistical computing. Vienna, Austria. https://www.R-project.org/. 
Roza, G., y Touzé, G. (2011). Las prácticas pre-profesionales en el contexto actual., Tensiones y nuevos desafíos. Revista "Debate Público. Reflexión de Trabajo Social”. Debates de Cátedra. Universidad de Buenos Aires, Argentina. p. 103-108.

Ruíz, M. A. (2019). Caracterización de 170 unidades de producción con enfoque agroecológico en nueve municipios del norte de Nicaragua. 2017-2018. Tesis de posgrado, Universidad Nacional Agraria, Mangua, Nicaragua.

Salas-Bourgoin, M. A. (2012). Propuesta de Índice de Calidad de Vida en la Vivienda. Cuadernos CENDES. Centro de Estudios del desarrollo. Universidad Central de Los Andes, 29(79). p. 57-78.

Sevilla Lucero, G., y Pila Semblantes, G. G. (2012). Elaboración de una guía de concientización sobre la importancia del laboratorio de práctica docente para las prácticas pre-profesionales en la carrera de educación básica en la Universidad Técnica de Cotopaxi en el periodo 2011-2012. Unidad académica de ciencias administrativas y humanísticas. UTC. 117 p.

Universidad Centroamericana. (2010). Problemática de la pobreza en Nicaragua. http://aulaweb.uca.edu.ni /blogs/edlacayo/ files/2010/08/Pobreza-de-Nic.pdf.

Verdejo, M. E. (2003). Diagnóstico rural participativo. Una guía práctica. Santo Domingo, República Dominicana. 118. p. 